\section{Ideas autolíticas, violencia autoinfligida, y síntomas depresivos en escolares chilenos}

\author{
SERGIO BARROILHET ${ }^{1,2}$, ROSEMARIE FRITSCH ${ }^{6,3}$, \\ VIVIANA GUAJARDO ${ }^{a}$, VANIA MARTÍNEZ ${ }^{4}$, PAUL VÖHRINGER ${ }^{2,3}$, \\ RICARDO ARAYA ${ }^{5}$, GRACIELA ROJAS ${ }^{3}$
}

\section{Suicidal ideation, self-directed violence and depression among Chilean school adolescents}

Background: Suicidal behaviors and depression are prevalent phenomena among adolescents, and are considered a public health problem. Aim: To determine the prevalence of depressive symptoms and suicidal behaviors and the relationship between both phenomena, in a representative sample of students from ninth grade in Santiago, Chile. Material and Methods: We recruited a probability sample of 2,597 adolescents who answered a questionnaire with questions about suicidal behavior and the Beck Depression Inventory (BDI-II). Results: The lifetime prevalence of suicidal ideation and planning was 21 and 14\%, respectively. The prevalence for the past two weeks was 6.7 and 4.4\% for suicidal ideation and planning, respectively. Autolytic behaviors, once in lifetime and in the past week were referred by 26 and $4 \%$ of respondents, respectively. In one third of these, self-harm coincided with recent suicide ideation or planning. All levels of suicidal behavior were more frequently reported by women. Clinically significant depressive symptoms were present in $23.5 \%$ of adolescents. Females doubled male rates. Severe depressive symptoms were present in $9.4 \%$ of the sample. A higher level of suicidal behavior correlated with more severe forms of depression. Sixty percent of adolescents who reported recent self-harm, had clinically relevant depressive symptoms. Two thirds of them had severe symptoms. Conclusions: Suicidal behavior in Chilean adolescents is prevalent, and there is an association between this behavior and the level of depression. The school is a good place to identify and develop preventive measures for teenagers.

(Rev Med Chile 2012; 140: 873-881).

Key words: Adolescent; Depression; Suicide, attempted; Suicidal ideation.

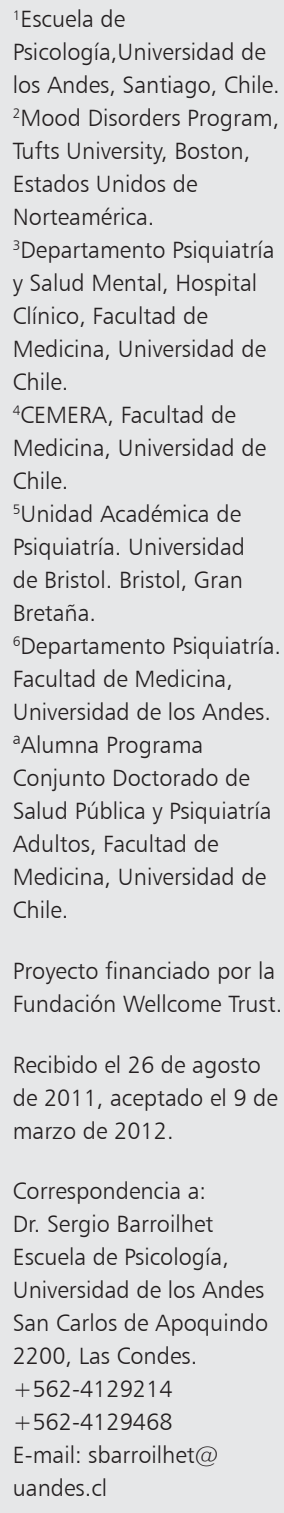

$\mathrm{E}$ n jóvenes, la depresión y conductas suicidales o autolíticas son un creciente problema social y de salud pública ${ }^{1}$. En el mundo, 4 millones de adolescentes intentan suicidarse anualmente, y alrededor de 100.000 mueren por esta causa ${ }^{1,2}$. El suicidio es la segunda causa de muerte entre 10 y 24 años en algunos países ${ }^{3}$, y entre los 15 y 19 años se encuentra entre las primeras cinco causas de mortalidad a nivel munidal ${ }^{4}$.

Chile duplica la tasa de mortalidad juvenil por suicidio de Latinoamérica y el Caribe ${ }^{5}$. Desde el año 2000 al 2005 ha aumentado en forma sostenida en los adolescentes ${ }^{6,7}$, siendo la segunda o tercera causa de muerte entre 15 y 19 años ${ }^{8}$.

El suicidio es la forma más extrema de violencia autoinfligida. Abarca un espectro de severidad, desde la desesperanza hasta el suicidio consumado, pasando por diversas formas de suicidalidad como ideación y conductas autoagresivas o autolíticas ${ }^{9,10}$. Según una revisión sistemática de suicidalidad adolescente, $30 \%$ presentaría ideación y $10 \%$ habría realizado un intento suicida a lo largo de la 
vida. Durante el último año, 19\% habría pensado en quitarse la vida, y $6 \%$ habría realizado algún intento. Excepto el suicidio consumado, todas las formas de suicidalidad son más prevalentes en adolescentes mujeres ${ }^{11}$. En Chile también es más frecuente en mujeres y en etapas más tardías de la adolescencia $^{12,13}$. Sin embargo, es difícil comparar los resultados debido a variaciones respecto de las muestras y metodología empleada. Según la Encuesta Mundial de Salud Escolar, la prevalencia de suicidalidad en escolares chilenos es la más alta entre varios países con $20-22 \%$ para ideación suicida entre $7^{\circ}$ básico y $1^{\circ}$ medio en el último año, y $15-17 \%$ de planificación ${ }^{14}$. Otros estudios chilenos muestran altas prevalencias de suicidalidad en áreas urbanas y rurales a lo largo de la vida en jóvenes ${ }^{12,15-17}$. Se ha encontrado una prevalencia de vida de $57 \%$ para ideas en torno a la muerte y suicidales, y $14,2 \%$ para intento suicida. La prevalencia de intento suicida para el último año fue $7,2 \%{ }^{17}$. En un estudio subsecuente, $43,3 \%$ de los adolescentes presentó ideación suicida el último año, y $19,1 \%$ habría intentado ${ }^{12}$. En la Región Metropolitana la prevalencia para el último año de ideación suicida fue $36,1 \%$, y $19 \%$ para intento de suicidio. Ambos son mayores entre mujeres de 16 años de escuelas públicas ${ }^{13}$. No hay datos sobre prevalencia de planificación suicida. Del total de egresos hospitalarios de adolescentes entre 12 y 19 años entre el 2002 y 2007, 0,66\% recibió un diagnóstico de daño autoinfligido, y $0,89 \%$ de daño de intención no determinada. Dos por ciento y $2,1 \%$, respectivamente, egresaron fallecidos ${ }^{7}$.

La depresión constituye un factor de riesgo suicida $^{18,19}$, presente en 49 a $64 \%$ de los adolescentes muertos por suicidio ${ }^{20}$. En Chile, la prevalencia actual de episodio depresivo es 5\% en población general mayor de 15 años ${ }^{21-23}$, siendo más frecuente en mujeres. En población general, la prevalencia actual de depresión es 5,8\% entre 15 y 24 años (Fritsch, 2011, comunicación verbal) en Santiago, y $5,1 \%$ para trastornos afectivos entre 12 y 18 años ${ }^{23}$. Sin embargo, la prevalencia es mayor al utilizar escalas para medir severidad de sintomatología depresiva o depresividad. Entre 12 y 20 años estaría entre $13,2 \%{ }^{24}$ y $44 \%^{25}$, con predomino en mujeres.

$\mathrm{Si}$ bien está bien estudiado que el aumento en la severidad de la depresividad es un factor de riesgo de suicidalidad ${ }^{26,27}$, se sabe menos acerca de cuál es la prevalencia de sintomatología depresiva en los distintos niveles de suicidalidad. Esto es particularmente interesante en adolescentes dada la creciente consulta por estos motivos en los servicios especializado ${ }^{28-30}$, y las presuntas diferencias en comparación con adultos ${ }^{9,31}$. En una muestra de estudiantes secundarios se encontró que del $5,6 \%$ que había presentado algún intento suicida en el último año, el 60\% presentaba depresividad clínicamente significativa ${ }^{32}$.

El objetivo del presente trabajo es determinar la prevalencia y severidad de síntomas depresivos y suicidalidad, y la relación entre ambos fenómenos en una muestra representativa de adolescentes escolares del Gran Santiago.

\section{Metodología}

El estudio es parte del diagnóstico basal del ensayo clínico aleatorio "Intervención Escolar para mejorar la Salud Mental de jóvenes de Enseñanza Media" ${ }^{33}$, llevado a cabo entre marzo y abril de 2009 en una muestra probabilística de adolescentes pertenecientes a establecimientos educacionales municipales. Fue aprobado por el Comité de Ética del Hospital Clínico de la Universidad de Chile.

\section{Muestra}

Se seleccionaron aleatoriamente 23 establecimientos municipalizados del Gran Santiago, de un universo de 95 establecimientos que cumplían con los criterios de inclusión (mixtos y con al menos 2 cursos de $1^{\circ}$ Año de Enseñanza Media) $)^{34}$. Se excluyeron aquellos que impartieran educación especial, a adultos, o vespertina. Fueron estratificados según estrato socioeconómico, utilizando el Î́ndice de Vulnerabilidad del Establecimiento (IVE) ${ }^{35}$ y el tamaño del establecimiento. Se buscó que todos los estratos estuvieran igualmente representados en la muestra seleccionada.

\section{Evaluación}

Se evaluó a los estudiantes en el aula. La participación fue voluntaria, previo consentimiento escrito de los padres y asentimiento de los estudiantes. Este procedimiento fue conducido por 22 evaluadores seleccionados que recibieron 6 horas de entrenamiento. Se logró evaluar a la totalidad de la muestra entre marzo y abril de 2009.

\section{Instrumentos}

Se administró un cuestionario autoaplicado 
con preguntas sobre datos sociodemográficos, suicidalidad y comportamientos autolíticos. Éste fue elaborado en base a cuestionarios previamente utilizados en Europa ${ }^{36,37}$ (ver Anexo 1), y evalúa prevalencia de pensamientos y conductas suicidas o autoagresivos en distintos momentos, abarcando prevalencias de vida y actuales. Se evaluó la severidad de los síntomas depresivos mediante el Inventario de Depresividad de Beck (BDI-II), el cual contiene 21 ítems y ha sido validado en adolescentes chilenos ${ }^{16,38}$. Su puntaje va de 0 a 63 puntos. Puede categorizarse en 4 tramos de severidad: depresividad mínima o ausente (0-13 pts); depresividad leve (14-19 pts); moderada (20-28 pts); y severa (29-63 pts). El punto de corte para establecer la presencia de depresividad clínicamente significativa en adolescentes chilenos estaría en 19 puntos $^{38}$.

\section{Análisis de los datos}

Para el análisis estadístico se utilizó la versión 11.0 del SPSS y STATA 8.0. Se realizaron análisis de frecuencia para variables cualitativas, y promedio e intervalo de confianza para las variables cuantitativas. En el análisis bivariado, las variables categóricas ordinales y nominales se analizaron mediante la prueba de $\chi^{2}$. Para la comparación de medias se utilizó el ANOVA (HSD Tukey). Se realizaron análisis mediante regresión logística con y sin ajuste, y se extrajeron las razones de verosimilitud y sus intervalos de confianza.

\section{Resultados}

\section{Descripción de la muestra}

La muestra estuvo constituida por 2.597 escolares, 1.157 mujeres (44,6\%; IC 95\%: 42,6-46,4) y 1.440 hombres (55,4\%; IC 95\%: 53,6-57,4), de 69 primeros medios pertenecientes a 23 establecimientos escolares, con una edad promedio de 15,1 años (IC 95\%: 15,0-15,2; rango: 13-19 años).

\section{Suicidalidad}

La prevalencia de los distintos niveles de suicidalidad aparece en la Tabla 1. Durante su vida, una proporción importante de los jóvenes ha presentado ideación suicida alguna vez. La mayoría de ellos refirió haber llegado a planificarlo. Uno de cada cuatro refirió presencia de ideación durante los úl-

Tabla 1. Prevalencia de niveles de gravedad de la suicidalidad por sexo $(n=2.597)$

\begin{tabular}{|c|c|c|c|c|}
\hline \multirow[b]{2}{*}{ Gravedad de suicidalidad } & $\begin{array}{l}\text { Total casos } \\
\text { válidos }\end{array}$ & Hombres & Mujeres & \multirow[b]{2}{*}{$\begin{array}{c}\text { OR ajustado } \\
\text { (IC95\%) }\end{array}$} \\
\hline & n (\%) & n (\%) & n (\%) & \\
\hline \multicolumn{5}{|l|}{ Desesperanza } \\
\hline Últimos 14 días & $552(20,1)$ & $214(14,9)$ & $308(26,7)$ & $2,2(1,8-2,6)^{* *}$ \\
\hline \multicolumn{5}{|l|}{ Vida no vale la pena } \\
\hline Últimos 14 días & $438(16,9)$ & $159(11,0)$ & $279(24,1)$ & $2,6(2,1-3,3) * *$ \\
\hline \multicolumn{5}{|l|}{ Ideación suicida } \\
\hline Últimos 14 días & $174(6,7)$ & $66(4,6)$ & $108(9,3)$ & $2,2(1,6-3,0) * *$ \\
\hline Alguna vez en la vida & $545(21,0)$ & $186(12,9)$ & $359(31,0)$ & $3,1(2,6-3,8) * *$ \\
\hline \multicolumn{5}{|l|}{ Planificación suicida } \\
\hline Últimos 14 días & $113(4,4)$ & $37(2,6)$ & $76(6,6)$ & $2,7(1,8-4,1) * *$ \\
\hline Ha hecho planes para quitarse la vida alguna vez & $365(14,1)$ & $112(7,8)$ & $253(21,9)$ & $3,5(2,7-4,4) * *$ \\
\hline \multicolumn{5}{|l|}{ Intentos suicidas o autoagresiones } \\
\hline Última semana & $101(3,9)$ & $42(2,9)$ & $59(5,1)$ & $1,8(1,2-2,7)^{*}$ \\
\hline Último año & $470(18,1)$ & $134(9,3)$ & $336(29,0)$ & $4,1(3,3-5,2) * *$ \\
\hline Alguna vez & $682(26,3)$ & $249(17,3)$ & $433(37,4)$ & $3,0(2,5-3,6) * *$ \\
\hline
\end{tabular}

${ }^{\dagger}$ modelo ajustado por edad. ${ }^{* *} p<0,001,{ }^{*} p<0,01$. 
Tabla 2. Prevalencia de sintomatología depresiva de acuerdo a gravedad de suicidalidad (BDI-II)

\begin{tabular}{|c|c|c|c|c|c|c|}
\hline Gravedad de suicidalidad & $\begin{array}{c}\text { Mínima o } \\
\text { ausente } \\
\text { n (\%) }\end{array}$ & $\begin{array}{l}\text { Leve } \\
\text { n (\%) }\end{array}$ & $\begin{array}{l}\text { Moderada } \\
\text { n (\%) }\end{array}$ & $\begin{array}{l}\text { Severa } \\
\text { n (\%) }\end{array}$ & $\begin{array}{l}\text { Total } \\
\text { n (\%) }\end{array}$ & Valor $\mathbf{p}$ \\
\hline $\begin{array}{l}\text { Desesperanza en últimos } 14 \text { días } \\
(\mathrm{n}=522)\end{array}$ & $115(22,0)^{\#}$ & $102(19,5)^{*}$ & $136(26,1)^{*}$ & $169(32,4)^{*}$ & $522(100)$ & $<0,001$ \\
\hline $\begin{array}{l}\text { Vida no vale la pena últimos } 14 \text { días } \\
(\mathrm{n}=438)\end{array}$ & $92(21,0)^{\#}$ & $82(18,7)$ & $104(23,7)^{*}$ & $160(36,5)^{*}$ & $438(100)$ & $<0,001$ \\
\hline $\begin{array}{l}\text { Ideación suicida últimos } 14 \text { días } \\
(\mathrm{n}=174)\end{array}$ & $35(20,1)^{\#}$ & $32(18,4)$ & $36(20,7)^{*}$ & $71(40,8)^{*}$ & $174(100)$ & $<0,001$ \\
\hline $\begin{array}{l}\text { Planificación suicida últimos } 14 \text { días } \\
(\mathrm{n}=113)\end{array}$ & $21(18,6)^{\#}$ & $23(20,4)$ & $19(16,8)$ & $50(44,2)^{*}$ & $113(100)$ & $<0,001$ \\
\hline $\begin{array}{l}\text { Intentos suicidas o autoagresiones } \\
\text { en la última semana }(n=101)\end{array}$ & $26(25,7)^{\#}$ & $14(13,9)^{\#}$ & $23(22,8)$ & $38(37,6)^{\#}$ & $101(100)$ & $<0,006$ \\
\hline
\end{tabular}

\#Menos casos de los esperados; * Más casos de los esperados.

timos 14 días. Tres coma nueve por ciento (IC95\%: $3,2-4,7)$ reportó conductas autolíticas durante la última semana, y $1 / 3$ de ellos presentaron ideación y/o planificación suicida concomitante en los últimos 14 días (1,3\%; IC95\%: 0,9-1,9). El 94,1\% $(\mathrm{n}=646)$ de los que refirieron conductas autolíticas describieron el método utilizado en la última oportunidad. Los más utilizados fueron: cortes en la piel $(56,2 \%)$, ingesta de medicamentos o veneno $(21,8 \%)$, quemarse la piel $(4,2 \%)$, u otros $(17,8 \%)$, como intentos de asfixia o ahorcamiento, golpear objetos sólidos en diferentes partes del cuerpo, o autoinfligirse golpes o heridas directamente. Entre los adolescentes con conductas autolíticas que presentaron ideación y/o planificación suicida en los últimos 14 días, predominaron los cortes $(45,7 \%)$ y la intoxicación con medicamentos o veneno $(31,4 \%)$. Las mujeres refirieron mayor frecuencia de haber ingerido pastillas o veneno $(\mathrm{p}<0,001)$. Encontramos una asociación significativa de todos los parámetros de suicidalidad con la edad y el sexo de los entrevistados $(\mathrm{p}<0,001)$. Las mujeres presentaron mayores prevalencias de suicidalidad, $\mathrm{y}$ al ajustar por edad, presentaron 2 a 4 veces más riesgo de ésta en sus diversos grados en comparación con sus pares de sexo masculino.

\section{Intensidad de la sintomatología depresiva}

La muestra total obtuvo un puntaje promedio en el BDI-II de 13,4 puntos (IC 95\%: 13,0-13,8); el promedio en las mujeres fue mayor que en los hombres (16,7 puntos (IC 95\%: 16,1-17,3; Me- diana: 15,0 ) vs 10,7 puntos (IC 95\%: 10,3-11,2; Mediana: 9,0); U de Mann-Whitney; $\mathrm{p}<0,001$ ).

La prevalencia de sintomatología depresiva clínicamente significativa fue de 23,5\% (IC 95\%: $21,8-25,1)$ para el total de la muestra, afectando al $34,4 \%$ de las mujeres (IC 95\%: 31,6-37,2) en comparación con el $14,7 \%$ de los hombres (IC 95\%: 12,9-16,6) $(\mathrm{p}<0,001)$.

El 9,4\% (IC 95\%: 8,3-10,6) de los jóvenes presentaron depresividad de carácter grave, $14,0 \%$ (IC 95\%: 12,7-15,4) de carácter moderado, y 16,4\% (IC 95\%: 14,0-17,8) de carácter leve.

Encontramos asociación entre la intensidad de la depresividad con la edad ( $\mathrm{p}<0,001)$ y el sexo $(\mathrm{p}<0,001)$. Las mujeres presentaron mayor frecuencia de sintomatología depresiva, en todas las categorías de intensidad. Al ajustar por edad, el sexo femenino se asoció a un riesgo creciente de depresividad de mayor severidad, llegando a cuadriplicar el riesgo de depresividad severa (OR: 3,8 IC 95\%: 2,9-5,1).

\section{Los pensamientos y conductas suicidas, y la} intensidad de la sintomatología depresiva

A mayor nivel de suicidalidad fueron progresivamente más frecuentes formas más severas de depresividad ( $r h o=0,477 ; \mathrm{p}<0,001)$ (Tabla 2). Esta asociación se mantuvo al ajustar por edad y sexo (Tabla 3). Las mujeres presentan mayores niveles de depresividad que los hombres en todos los niveles de suicidalidad. Estos mayores niveles de depresividad son a niveles menores de suicida- 
Tabla 3. Riesgo de severidad de la sintomatología depresiva según nivel de gravedad de la suicidalidad

\begin{tabular}{|c|c|c|c|c|c|c|c|c|c|c|}
\hline \multirow[b]{3}{*}{$\begin{array}{l}\text { Depresi- } \\
\text { vidad }\end{array}$} & \multicolumn{10}{|c|}{ Gravedad de suicidalidad } \\
\hline & \multicolumn{2}{|c|}{$\begin{array}{l}\text { Desesperanza } \\
\text { en últimos } 14 \text { días } \\
(n=522)\end{array}$} & \multicolumn{2}{|c|}{$\begin{array}{c}\text { Sin deseos } \\
\text { de vivir últimos } \\
14 \text { días } \\
(n=438)\end{array}$} & \multicolumn{2}{|c|}{$\begin{array}{l}\text { Ideación suicida } \\
\text { últimos } 14 \text { días } \\
\text { (n=174) }\end{array}$} & \multicolumn{2}{|c|}{$\begin{array}{l}\text { Planificación } \\
\text { suicida últimos } \\
14 \text { días } \\
(n=113)\end{array}$} & \multicolumn{2}{|c|}{$\begin{array}{c}\text { Intentos suicidas o } \\
\text { autoagresiones en } \\
\text { la última semana } \\
(\mathbf{n}=101)\end{array}$} \\
\hline & $\begin{array}{c}\text { OR } \\
\text { (IC95\%) } \\
\text { crudo }\end{array}$ & $\begin{array}{c}\text { OR } \\
\text { (IC95\%) } \\
\text { ajustado }\end{array}$ & $\begin{array}{c}\text { OR } \\
\text { (IC95\%) } \\
\text { crudo }\end{array}$ & $\begin{array}{c}\text { OR } \\
\text { (IC95\%) } \\
\text { ajustado }\end{array}$ & $\begin{array}{c}\text { OR } \\
\text { (IC95\%) } \\
\text { crudo }\end{array}$ & $\begin{array}{c}\text { OR } \\
\text { (IC95\%) } \\
\text { ajustado }\end{array}$ & $\begin{array}{c}\text { OR } \\
\text { (IC95\%) } \\
\text { crudo }\end{array}$ & $\begin{array}{c}\text { OR } \\
\text { (IC95\%) } \\
\text { ajustado }\end{array}$ & $\begin{array}{c}\text { OR } \\
\text { (IC95\%) } \\
\text { crudo }\end{array}$ & $\begin{array}{c}\text { OR } \\
\text { (IC95\%) } \\
\text { ajustado }\end{array}$ \\
\hline 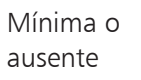 & 1 & 1 & 1 & 1 & 1 & 1 & 1 & 1 & 1 & 1 \\
\hline Leve & $\begin{array}{c}4,0 \\
(3,0-5,36)^{* *}\end{array}$ & $\begin{array}{c}3,9 \\
(2,9-5,24)^{* *}\end{array}$ & $\begin{array}{c}3,8 \\
(2,8-5,3)^{* *}\end{array}$ & $\begin{array}{c}3,5 \\
(2,5-4,9)^{* *}\end{array}$ & $\begin{array}{c}3,6 \\
(2,2-5,8)^{* *}\end{array}$ & $\begin{array}{c}3,3 \\
(2,0-5,5)^{* *}\end{array}$ & $\begin{array}{c}4,2 \\
(2,3-7,7)^{* *}\end{array}$ & $\begin{array}{c}3,6 \\
(2,0-6,7)^{* *}\end{array}$ & $\begin{array}{c}2,0 \\
(1,0-3,9)^{*}\end{array}$ & $\begin{array}{c}1,8 \\
(0,9-3,6)\end{array}$ \\
\hline Moderada & $\begin{array}{c}7,5 \\
(5,7-10,0)^{* *}\end{array}$ & $\begin{array}{c}7,3 \\
(5,4-9,8)^{* *}\end{array}$ & $\begin{array}{c}6,4 \\
(4,7-8,8)^{* *}\end{array}$ & $\begin{array}{c}5,8 \\
(4,2-7,9)^{* *}\end{array}$ & $\begin{array}{c}4,8 \\
(3,0-7,8)^{* *}\end{array}$ & $\begin{array}{c}4,4 \\
(2,7-7,3)^{*+*}\end{array}$ & $\begin{array}{c}4,1 \\
(2,2-7,6)^{* *}\end{array}$ & $\begin{array}{c}3,4 \\
(1,8-6,4)^{* *}\end{array}$ & $\begin{array}{c}4,0 \\
(2,2-7,1)^{* *}\end{array}$ & $\begin{array}{c}3,8 \\
(2,1-6,8)^{* *}\end{array}$ \\
\hline Severa & $\begin{array}{c}28,3 \\
(20,3-39-5)^{* *}\end{array}$ & $\begin{array}{c}26,9 \\
19,1-38,0)^{* *}\end{array}$ & $\begin{array}{c}30,7 \\
(21,9-43,1)^{* *}\end{array}$ & $\begin{array}{c}26,9 \\
(18,9-38,2)^{* *}\end{array}$ & $\begin{array}{c}17,9 \\
(11,6-27,6)^{* *}\end{array}$ & $\begin{array}{c}16,2 \\
(10,2-25,5)^{* *}\end{array}$ & $\begin{array}{c}18,9 \\
(11,1-32,2)^{* *}\end{array}$ & $\begin{array}{c}15,1 \\
(8,6-26,3)^{* * *}\end{array}$ & $\begin{array}{c}10,9 \\
(6,5-18,2)^{* *}\end{array}$ & $\begin{array}{c}10,1 \\
(5,9-17,6)^{* *}\end{array}$ \\
\hline
\end{tabular}

${ }^{* *} p<0,001 ;{ }^{*} p<0,05$.

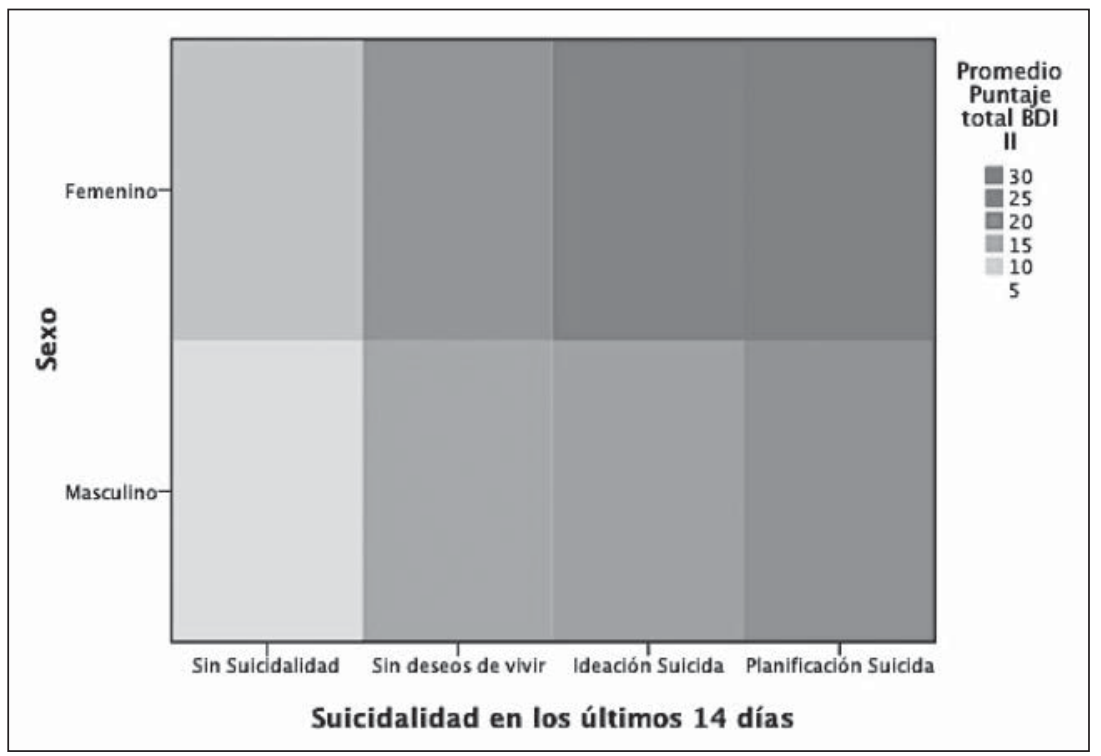

Figura 1. Depresividad asociada a los distintos niveles de gravedad de la suicidalidad según sexo. lidad, en comparación con hombres (Figura 1).

De los adolescentes que refirieron autoagresiones en la última semana, el 60,4\% (equivalente al $2,3 \%$ del total) presentó depresividad clínicamente signficativa, y aproximadamente $2 / 3$ de ellos presentaron la mayor severidad (1,5\%; IC $95 \%$ : $1,0-2,0)$.

Los adolescentes que presentaron conductas autolíticas en la última semana coincidiendo con ideación suicida en las últimas dos semanas, pre- sentaron depresividad moderada (Media: 23,9 pts; IC 95\%: 12,6-35,1). Aquellos que presentaron estas conductas coincidiendo con planificación suicida en el mismo período presentaron una intesisdad severa (Media: 30,5; IC 95\%: 24,2-36,7). Los que no presentaron estas conductas en la última semana, en promedio no presentaron depresividad (Media: 13,0; IC 95\%: 12,6-13,4). En los contrastes post-hoc se encontraron diferencias significativas entre los puntajes de los adolescentes con y sin 
autoagresiones; y entre conductas autolíticas sin ideación y/o planificación suicida, y las con planificación (F: 43,591; p < 0,001; Tukey HSD < 0,001).

Aquellos adolescentes con conductas autolíticas sin ideación ni planificación suicida $(2,5 \%$; IC 95\%: 2,0-3,2) igualmente presentaron depresividad moderada (Media: 21,3 pts; IC 95\%: 18,2-24,3).

\section{Discusión}

Encontramos una alta prevalencia de adolescentes que alguna vez en la vida presentaron ideación y/o planificicación suicida. Esto es concordante los resultados de la Encuesta Mundial de Salud Escolar en Chile, repitiéndose la mayor frecuencia para las adolescentes de sexo femenino. Este dato confirma la alta prevalencia en Chile en comparación con otros países ${ }^{14}$. No obstante, al comparar con otros estudios realizados con escalas específicas $^{12,13,17}$ en adolescentes chilenos, nuestros resultados son inferiores, lo cual podría explicarse por diferencias muestrales y metodológicas. Destaca que uno de cada 100 adolescentes presentaría conductas autolíticas concomitantemente con ideación y/o planificación suicida.

Estudios señalan que hasta $40 \%$ de los adolescentes que se autoagreden tienen pensamientos suicidas durante la autoagresión ${ }^{39}$. Si bien dadas las tasas actuales en Chile al parecer pocos de estos últimos llegan a suicidarse, cabe considerar que los suicidios adolescentes pudieran ser subestima$\operatorname{dos}^{40}$. Se desconoce si algunas muertes accidentales pudieran ser suicidios no diagnosticados ${ }^{9}$. Pudiera orientar el hecho de que la frecuencia de egresos hospitaliarios de adolescentes debido a muerte por lesiones de intencionalidad no determinada es equivalente a la de egresos por muerte secundaria a daños autoinfligidos ${ }^{7}$. No obstante no existen cifras oficiales acerca de cuántos adolescentes consultan en los servicios de urgencia por intentos autolíticos, y cuáles son los costos asociados en términos de morbilidad.

La suicidalidad es un fenómeno complejo, en el cual intervienen diversos factores psicológicos, sociales, biológicos, culturales y medioambientales ${ }^{9}$. Inicialmente tener pensamientos suicidas puede ser parte de un proceso normal de desarrollo adolescente $e^{4,31,41}$. La presencia de estas ideas pudiera estar relacionada con el atravesar por contextos psicosociales difíciles durante la etapa adolescente ${ }^{12}$. Los pensamientos suicidas se volverían patológicos cuando la materialización de estos pensamientos aparece como la única salida para las dificultades vivenciadas ${ }^{4}$. En efecto, en Chile hay evidencias de mayor prevalencia de suicidalidad a menor nivel socioeconómico ${ }^{13,42}$.

También encontramos una alta prevalencia de depresividad en la población estudiada, concordante con estudios previos ${ }^{16,24,25}$. Al igual que en adultos, la presencia depresividad clínicamente significativa es un factor de riesgo de suicidalidad en adolescentes ${ }^{9}$, sin embargo, esta relación no está suficientemente estudiada en Chile. Concordante con estudios previos ${ }^{32}$, los síntomas depresivos resultaron frecuentes en adolescentes suicidales. Frente a un adolescente en que se pesquisa algún grado de suicidalidad hay entre 10 y 30 veces mayor riesgo de depresividad grave.

La severidad de la depresividad es distinta entre adolescentes que se autoagredieron sólo con ideación, versus aquellos que lo hicieron con planificación. Frente a la alta prevalencia de ideación suicida entre los adolescentes chilenos, determinar la presencia de planificación podría agregar especificidad y delimitar mejor la población en riesgo. En estudios previos no se ha realizado esta distinción ${ }^{13,15,17,32}$.

Encontramos que las autoagresiones sin ideación o planificación suicida, se asociaron a depresividad clínicamente significativa. Las autoagresiones no suicidas tendrían numerosas funciones como aliviar emociones negativas ${ }^{39}$ como angustia, culpa, soledad, alienación u odio contra sí mismo; liberar la ira, tensión o dolor emocional; restaurar sentimientos de seguridad o control; autocastigo; poner límites o llamar la atención de otros; terminar con estados de despersonalización/desrrealización, flashbacks, o pensamiento acelerado; o varias de ellas simultáneamente ${ }^{43}$. Pero también pudieran ser un reflejo de una menor capacidad de dar cuenta de sus procesos internos ${ }^{44}$. Todo esto pudiera asociarse a una incapacidad para afrontar la situación vital del momento, lo cual pudiera traducir una baja capacidad de solución de problemas ${ }^{45}$. En cualquier caso, la detección de autoagresiones no suicidas tendría relevancia clínica: podría ser un marcador clínico de depresividad, y podría solaparse en el tiempo con comportamientos suicidas ${ }^{46}$. Aproximadamente 50 a $85 \%$ de las personas que se autoagreden realizan un intento suicida al menos una vez en la vida ${ }^{47}$. Por ende las autoagresiones no suicidas serían un 
factor de riesgo de suicidio ${ }^{48}$, añadiéndose al riesgo conferido por el estado depresivo.

Consecuentemente se hace necesario implementar estrategias preventivas eficaces que per- mitan revertir las tendencias actuales en cuanto a suicidalidad en Chile. Las escuelas aparecen como un lugar excelente para desarrollar acciones preventivas apropiadas ${ }^{4,41}$.

\section{Anexo 1. Cuestionario de suicidalidad y comportamientos autolíticos}

\begin{tabular}{|c|c|}
\hline Ítem & Respuesta \\
\hline $\begin{array}{l}\text { Durante los ÚLTIMOS } 14 \text { DÍAS, ¿Te has sentido sin esperanza en relación } \\
\text { al futuro? }\end{array}$ & $\begin{array}{l}\mathrm{SI} \\
\mathrm{NO}\end{array}$ \\
\hline $\begin{array}{l}\text { ¿Has pensado que no vale la pena seguir viviendo en los ÚLTIMOS } 14 \\
\text { DÍAS? }\end{array}$ & $\begin{array}{l}\mathrm{SI} \\
\mathrm{NO}\end{array}$ \\
\hline ¿Has pensado en quitarte la vida en los ÚLTIMOS 14 DÍAS? & $\begin{array}{l}\text { SI } \\
\text { Sí, pero no en los últimos } 14 \text { días } \\
\text { NO }\end{array}$ \\
\hline ¿Has hecho planes de cómo quitarte la vida EN LOS ÚLTIMOS 14 DÍAS? & $\begin{array}{l}\text { SI } \\
\text { Sí, pero no en los últimos } 14 \text { días } \\
\text { NO }\end{array}$ \\
\hline $\begin{array}{l}\text { Has intentado alguna vez quitarte la vida o hacerte daño o auto-agre- } \\
\text { dirte de alguna forma, como por ejemplo tomando una sobredosis de } \\
\text { medicamentos o cortándote los brazos? }\end{array}$ & $\begin{array}{l}\mathrm{SI} \\
\mathrm{NO}\end{array}$ \\
\hline $\begin{array}{l}\text { ¿Cuándo fue la última vez que te hiciste daño a propósito, o te au- } \\
\text { toagrediste? }\end{array}$ & $\begin{array}{l}\text { En la última semana } \\
\text { Hace más de una semana pero en el último año } \\
\text { Hace más de un año }\end{array}$ \\
\hline $\begin{array}{l}\text { De la última vez que te autoagrediste a propósito, marca lo que mejor } \\
\text { describe lo que hiciste. Puedes marcar todas las casillas que corres- } \\
\text { pondan }\end{array}$ & $\begin{array}{l}\text { Ingerir pastillas o algún veneno } \\
\text { Me hice cortes } \\
\text { Me quemé, por ejemplo, con cigarros } \\
\text { Otro (explica qué hiciste) }\end{array}$ \\
\hline
\end{tabular}

\section{Referencias}

1. Organización Mundial de la Salud. Orientaciones estratégicas para mejorar la salud y el desarrollo de los niños y los adolescentes. p. 6. Disponible en: http://www.who. int/child_adolescent_health/documents/WHO_FCH_ CAH_02.21/es/index.html [Consultado el 16 de agosto de 2011].

2. UNICEF. Adolescencia: Una etapa fundamental. Nueva York: UNICEFF; 2002. p.35. Disponible en: http://www. unicef.org/spanish/publications/index_4266.html [Consultado el 17 de agosto de 2011].

3. World Health Organization, Suicide Prevention Program (SUPRE), Disponible en:. http://www.who.int/ mental_health/prevention/suicide/suicideprevent/en/ [Consultado el 2 de agosto de 2011].

4. Bertolote JL. Prevención del suicidio: un instrumento para docentes y demás personal institucional. En: Preventing Suicide: a resource series. Ginebra: Organización Mundial de la Salud; 2001. Disponible en: http://www. who.int/mental_health/resources/preventingsuicide/en/ index.html [Consultado el 17 de agosto de 2011].

5. Comisión Económica para América Latina y el Caribe (CEPAL). Juventud Iberoamericana: Tendencias y Urgencias. Buenos Aires: CEPAL; 2007.

6. Ministerio de Salud de Chile. Objetivo Estratégico №4. Reducir la mortalidad, morbilidad y mejorar la salud de las personas, a lo largo de todo el ciclo vital. Santiago: MINSAL; 2010. p 108. Disponible en: http://www.redsalud.gov.cl/portal/url/item/94b4cb1b29bc26a2e0400101 1e013430.pdf [Consultado el 11 de agosto de 2011].

7. Deis-MINSAL, 2008. Base de datos descargable. Disponible en: http://deis.minsal.cl/deis/basededatos/bdd_descargables.asp [Consultado el 12 de agosto de 2011]. 
8. Valdivia M, Ebner D, Fierro V, Gajardo C, Miranda R. Hospitalización por intento de suicidio en población pediátrica: Una revisión de cuatro años. Rev Chil NeuroPsiquiatr 2001; 39: 211-8.

9. Oficina Regional para las Américas de la Organización Mundial de la Salud. Capítulo 7. La Violencia Autoinfligida. En: Informe mundial sobre la violencia y la salud. Washington, D.C.: Organización Panamericana de la Salud; 2003. p. 201-231. Disponible en: www.paho.org/ Spanish/AM/PUB/capitulo_7.pdf [Consultado el 10 de agosto de 2011].

10. Serfaty E. Suicidio en la adolescencia. Adolesc. Latinoam 1998; 1(2): 105-10.

11. Evans E, Hawton K, Rodham K, Deeks J. The Prevalence of Suicidal Phenomena in Adolescents: A Systematic Review of Population-Based Studies. Suicide Life Threat Behav 2005; 35 (3): 239-50.

12. Salvo L, Melipillán R. Predictores de suicidalidad en adolescentes. Rev Chil Neuro-Psiquiat 2008; 46 (2): 115-23.

13. Ventura-Juncá R, Carvajal C, Undurraga S, Vicuña $P$, Egaña J, Garib MJ. Prevalencia de Ideación e Intento Suicida en Adolescentes de la Región Metropolitana de Santiago de Chile. Rev Med Chile 2010; 138: 309-315.

14. Ministerio de Salud de Chile. Encuesta Mundial Salud Escolar, Chile 2004. Disponible en: http://epi.minsal.cl/ epi/html/invest/EMSE/INFORME\%20EMSE\%202004. pdf [Consultado el 4 de agosto de 2011].

15. Haquim C, Larraguibel M, Cabezas J. Factores protectores y de riesgo en salud mental en niños y adolescentes de la ciudad de Calama. Rev Chil Pediatr 2004; 75: 42533.

16. Cova F, Melipillán R, Valdivia M, Bravo E, Valenzuela B. Depresividad y ansiosa en estudiantes de enseñanza media. Rev Chil Pediatr 2007; 78: 151-9.

17. Salvo L, Rioseco P, Salvo S. Ideación suicida e intento de suicidio en adolescentes de enseñanza media. Rev Chil Neuro-Psiquiatr 1998; 36 (1): 29-35.

18. Blumenthal SJ. Suicide: a guide to risk factors, assessment, and treatment of suicidal patients. Med Clin North Am 1988; 72: 937-71.

19. Botswick JM, Pankratz VS. Affective disorders and suicide risk: a re-examination. Am J Psychiatry 2000; 157: 1925-32.

20. Steele MM, Doey T. Suicidal behaviour in children and adolescents. part 1: etiology and risk factors. Can J Psychiatry 2007; 52 (6 Suppl 1): 21S-33S.

21. Vicente PB, Rioseco P, Saldivia S, Kohn R, Torres S. Estudio chileno de prevalencia de patología psiquiátrica (DSM-III-R/CIDI) (ECPP). Rev Med Chile 2002; 13: 527-36.

22. Araya R, Rojas G, Fritsch R, Acuña J, Lewis G. Common mental disorders in Santiago, Chile: prevalence and socio-demographic correlates. Br J Psychiatry 2001; 178: 228-33.

23. Vicente B, De la Barra F, Saldivia S, Kohn R, Rioseco P, Melipillán R. Prevalence of child and adolescent psychiatric disorders in Santiago, Chile: a community epidemiological study Soc Psychiatry Psychatr Epidemiol 2011 Disponible en: http://www.springerlink.com/ content/427457h4u584j606/fulltext.pdf [Consultado el 17 de noviembre de 2011].

24. Gellona J, Zarraonandia A, Muñoz R, Flores M. Prevalencia de síntomas depresivos en escolares adolescentes del sector oriente de Santiago. Rev Psiquiatr Salud Ment 2005; 22 (1-2): 93-9.

25. Cumsille P, Martínez M. Síntomas de depresión en estudiantes de enseñanza media de Santiago. Rev Chil Pediatr 1997; 68: 74-7.

26. Casey PR, Dunn G, Kelly BD, Birkbeck G, Dalgard OS, Lehtinen V, et al. Factors associated with suicidal ideation in the general population: five-centre analysis from the ODIN study. Br J Psychiatry 2006; 189: 410-5.

27. Hintikka J, Koivumaa-Honkanen H, Lehto SM, Tolmunen T, Honkalampi K, Haatainen K, et al. Are factors associated with suicidal ideation true risk factors? A 3-year prospective follow-up study in a general population. Soc Psychiatry Psychiatr Epidemiol 2009; 44 (1): 29-33.

28. Peterson B, Zhang H, Santa Lucia R, Raymond B, King $\mathrm{R}$, Lewis M. Risk factors for presenting problems in child psychiatry emergencies. J Am Acad Child Adolesc Psychiatry 1996; 35: 1162-73.

29. Pacheco B, Lizana P, Celhay I, Pereira J. Características clínicas de niños y adolescentes menores de 18 años hospitalizados en una clínica psiquiátrica universitaria. Rev Med Chile 2007; 135: 751-8.

30. Valdivia M, Schaub C, Díaz M. Intento de suicidio en niños: algunos aspectos biodemográficos. Rev Chil Pediatr 1998; 2: 64-7.

31. Jellinek MS, Snyder JB. Depression and suicide in children and adolescents. Pediatr Rev 1998; 19 (8): 255-64.

32. Quinteros P, Grob F. Depresión y suicidalidad en una población no clínica de adolescentes. Bol Soc Psiquiat Neurol Infanc Adolesc 2003; 14: 4-8.

33. Araya R, Montgomery AA, Fritsch R, Gunnell D, Stallard $\mathrm{P}$, Noble $\mathrm{S}$, et al. School-based intervention to improve the mental health of low-income, secondary school students in Santiago, Chile (YPSA): study protocol for a randomized controlled trial. Trials. 2011; 19: 12:49 Disponible en: http://www.trialsjournal.com/content/12/1/49 [Consultado el 22 de diciembre de 2011].

34. Ministerio de Educación de Chile (MINEDUC) Matrícula 2008. [base de datos en Internet]. 2009. Disponible 
en: http://w3app.mineduc.cl/mineduc/ded/documentos/ Matricula_2008.zip. [Consultado el 17 de agosto de 2011].

35. Sistema Nacional de Asignación con Equidad para Becas JUNAEB (SINAE). Una nueva visión en la construcción de igualdad de oportunidades en la infancia. JUNAEB; 2005.

36. Madge N, Hewitt A, Hawton K, de Wilde EJ, Corcoran P, Fekete S, et al. Deliberate self-harm within an international community sample of young people: comparative findings from the Child \& Adolescent Self-harm in Europe (CASE) Study. J Child Psychol Psychiatry 2008; 49 (6): 667-77.

37. McManus S, Meltzer H, Brugha T, Bebbington P, Jenkins R. Adult psychiatric morbidity in England, 2007 Results of a household survey. Appendices and Glossary. Disponible en: http://www.ic.nhs.uk/statistics-anddata-collections/mental-health/mental-health-surveys/ adult-psychiatric-morbidity-in-england-2007-resultsof-a-household-survey [Consultado el 30 de noviembre de 2011].

38. Melipillán R, Cova F, Rincón P, Valdivia M. Propiedades psicométricas del Inventario de Depresión de Beck-II en adolescentes chilenos. Terapia Psicológica 2008; 26 (1): 59-69.

39. Kerr PL, Muehlenkamp JJ, Turner JM. Nonsuicidal selfinjury: a review of current research for family medicine and primary care physicians. J Am Board Fam Med 2010; 23 (2): 240-59.

40. Larraguibel M, González P, Martínez V, Valenzuela R. Factores de riesgo de la conducta suicida en niños y adolescentes. Rev Chil Pediatr 2000; 71(3): 183-91.

41. McKey PW, Jones RW, Barbe RH. Suicide and the School: a Practical Guide to Suicide Prevention. Horsham, PA: LRP Publications, 1993.

42. Moyano E, Barría R. Suicidio y producto interno bruto (PIB) en Chile: Hacia un modelo predictivo. Rev Latinoam Psicol 2006; 38 (2): 343-59.

43. Lloyd-Richardson EE, Perrine N, Dierker L, Kelley ML. Characteristics and functions of non-suicidal self-injury in a community sample of adolescents. Psychol Med 2007; 37 (8): 1183-92.

44. Oumaya M, Friedman S, Pham A, Abou Abdallah T, Guelfi JD, Rouillon F. Borderline personality disorder, self-mutilation and suicide: literature review. Encephale 2008; 34 (5): 452-8.

45. Nock MK, Mendes WB. Physiological arousal, distress tolerance, and social problem-solving deficits among adolescent self-injurers. J Consult Clin Psychol 2008; 76 (1): 28-38.

46. Joiner TE, Conwell Y, Fitzpatrick KK, Witte TK, Schmidt NB, Berlim MT, et al. Four studies on how past and current suicidality relate even when 'Everything but the kitchen sink' is covaried. J Abnorm Psychol 2005; 114: 291-303.

47. Stanley B, Winchel R, Molco A, Simeon D, Stanley M. Suicide and the self-harm continuum: phenomenological and biochemical evidence. Int Rev Psychiatry 1992; 4: 149-55.

48. Wilkinson PO. Nonsuicidal self-injury: a clear marker for suicide risk. J Am Acad Child Adolesc Psychiatry 2011; 50 (8): 741-3. 